\title{
Physical origin of X-ray flares following GRBs
}

\author{
Bing Zhang \\ Department of Physics, University of Nevada Las Vegas, Las Vegas, NV 89154
}

\begin{abstract}
One of the major achievements of Swift is the discovery of the erratic X-ray flares harboring nearly half of gamma-ray bursts (GRBs), both for long-duration and short-duration categories, and both for traditional hard GRBs and soft X-ray flashes (XRFs). Here I review the arguments in support of the suggestion that they are powered by reactivation of the GRB central engine, and that the emission site is typically "internal", i.e. at a distance within the forward shock front. The curvature effect that characterizes the decaying lightcurve slope during the fading phase of the flares provides an important clue. I will then discuss several suggestions to re-start the GRB central engine and comment on how future observations may help to unveil the physical origin of X-ray flares.
\end{abstract}

\section{INTRODUCTION}

The successful launch and operation of the Swift satellite offers the opportunity to unveil the final gap between the prompt emission and the late afterglow. With the co-operation of the Burst Alert Telescope (BAT) and the X-Ray Telescope (XRT), a canonical early X-ray afterglow lightcurve is emerging[1, 2, 3, 4], which includes five components beside the prompt emission itself (Fig.1), a steep decay component, a shallower-than-normal decay component, a normal decay component, a possible postjet-break steep decay component, as well as one or more erratic X-ray flares. Not every segment exists in every burst, but all the lightcurves could be in principle understood within such a general framework. These lightcurves bring invaluable information to understand prompt emission - afterglow transition, GRB emission site, central engine activity, forward-reverse shock physics, and GRB immediate environment (for a full discussion, see Ref. [2]).

The most interesting component is erratic X-ray flares detected in nearly half Swift GRBs[5, 6, 7, 4, 8]. The general observational properties of these flares include (e.g. [9]): (1) flares typically have rapid rise and fall times, with $\delta t / t_{\text {peak }} \ll 1$; (2) many light curves have evidence for an underlying afterglow power law component with the same slope; (3) in many bursts, multiple early flares exist in a same burst; (4) in some cases, e.g. GRB 050502B [5, 6], the flux increases are very large (factors of tens to hundreds); (5) flares soften as they progress; (6) the durations of the flares are positively correlated with the epochs when the flares happen; (7) in some cases, very late flares occur at around days after the trigger (e.g. GRBs 050502B [6] and 050724 [10, 11]; (8) there is no apparent difference between long-duration and short-duration GRBs and between normal, hard GRBs and soft XRFs as far as the X-ray flare properties are concerned. 


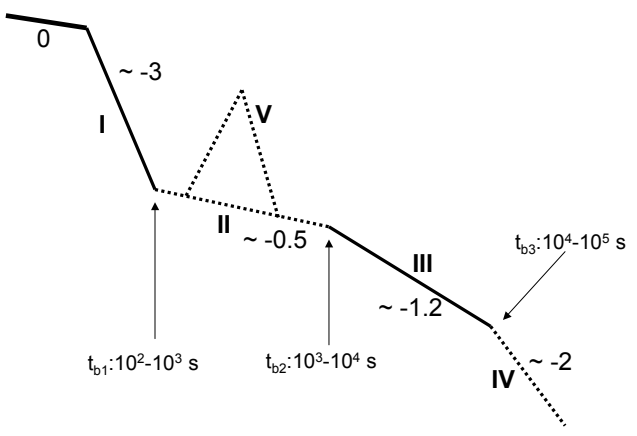

FIGURE 1. The canonical X-ray afterglow lightcurve. From Zhang et al. [2].

\section{X-RAY FLARE MECHANISM: LATE CENTRAL ENGINE ACTIVITY AND INTERNAL ENERGY DISSIPATION}

The data greatly constrained the possible models to interpret X-ray flares.

- The rapid rise and fall with $\delta t / t_{\text {peak }} \ll 1$ strongly disfavor the scenarios that invoke large angular scale external shock models [2, 12], including refreshed shocks, patchy-shell jets, multi-component jets, signatures induced by neutron decay, etc.

- The existence of multiple flares in a same GRB disfavors scenarios that can only account for one flare, e.g. the synchrotron self-Compton emission in the reverse shock [13], the deceleration of the blastwave [14], and the progenitor models invoking a companion[15].

- The comparable fluence (and hence, energy budget) of the giant X-ray flare of GRB 050502B [5, 6] disfavors models that interpret X-ray flares and prompt emission without additional energy input, including the clumpy medium model[2].

- The similar flaring behavior observed in both long and short bursts suggest that the flare mechanism is insensitive to the progenitor[16].

The leading model is that flares are due to the reactivation of the GRB central engine, and that they are produced from an "internal" dissipation radius within the external blastwave front [5, 2, 12, 17]. There are two advantages for such a model to interpret the flare data[2]. First, re-starting the GRB central engine effectively re-sets the startingtime. For example, should there be no prompt gamma-rays, the onset of a late flare would be effectively the "trigger" time. Each episode of the late central engine activity is equivalent to each other and should define its own time zero point $\left(t_{0}\right)$. Such a shift is essential to define the most relevant temporal decay index in the $\log F_{V}-\log \left(t-t_{0}\right)$ lightcurves, especially when $t$ is not much larger than $t_{0}$ (here all the numerical number of the time quantities are with respect to the trigger time). If $t_{0}$ is far from the trigger time, the conventional lightcurves ( $\log F_{V}-\log t$, with time zero point at the trigger time) 
would show artificial very steep decays as observed (sometimes the decay index after the flare peak is steeper than -7). Second, this model is very economical energetically. This is because we invoke two different emission sites to interpret the X-ray flares and the background power-law decaying X-ray afterglow. The former are from an internal radius, while the latter is from the external forward shock. In order to produce the same $\mathrm{X}$-ray flux level, the internal model usually only requires a small amount of energy budget with respect to that of the prompt emission, and the lightcurve including the prompt emission and the flares essentially reflects the time history of the central engine energy input. For the external shock model, on the other hand, one requires at least comparable energy budget with the intial blastwave in order to make any noticeable change of the afterglow flux level[18]. The flare amplitudes are usually a factor of several to several hundreds. This would require an unbelievably large energy input from the central engine without any observational signature. This is rather implausible. For external shock models that invoke very small density clumps[19], the energy budget problem could be eased, but so far no modeling could successfully reproduce the giant flares as observed in GRB 050502B.

Invoking an unknown central engine activity inevitably introduces difficulties to model the flares (as compared with the simply afterglow model). However, one has a clean signature that could be used to diagnose the above interpretation. This is the socalled "curvature effect" that dominates the decaying phase of the flare. Assuming that radiation stops abruptly at an internal radius from a conical shell, and that the cooling frequency is below the X-ray band before the cessation of the synchrotron emission, no fresh electrons would contribute to the emission in the X-ray band since the cessation of the emitter. What one detects is then the emission from higher latitudes with respect to the viewing direction that propagates to the observer with slight delay due to the extra distance the photons need to travel. There is a clear prediction for such a scenario: the temporal decay index is connected to the spectral index by $\alpha=\beta+2$ with the convention $F_{V} \propto v^{-\beta} t^{-\alpha}[20,21,2,17,22,23]$. Such a prediction is rather robust regardless of the speed history of the shell[2, 17] and is largely insensitive to the jet structure as long as the viewing angle is not very far away from the bright core of the structured jets[23].

The clean $\alpha=\beta+2$ characteristic is complicated by two additional effects [2]. One is the $t_{0}$ effect, i.e. one needs to identify the correct central engine starting time for the flare in investigation. If the internal-origin of the flares is correct, this $t_{0}$ should be at the beginning of the rising segment of the flare. The second effect is the superposition effect. There is an underlying afterglow level which tends to make the decay slope shallower, especially near the transition time between the steep decay component (segment I in Fig.1) and the shallow decay components from the afterglow (segments II or III in Fig.1). However, since both the spectral index $(\beta)$ and the temporal decay indices in different segments $\left(\alpha_{1}, \alpha_{2}\right.$ and $\left.\alpha_{3}\right)$ could be directly inferred from the data, one has a clean strategy to test the correctness of the internal-curvature interpretation.

Liang et al. [8] have performed such a systematic test. The procedure is the following. (1) Identify a steep decay component with $\alpha>2$ following a certain X-ray flare (or the steep decay component following the prompt emission). (2) Perform a fit to the afterglow lightcurve with two overlapping power laws. The late-time index is fitted by the data and the early-time index is assumed to be $\beta+2$. (3) Search for the time zero point $t_{0}$ that 

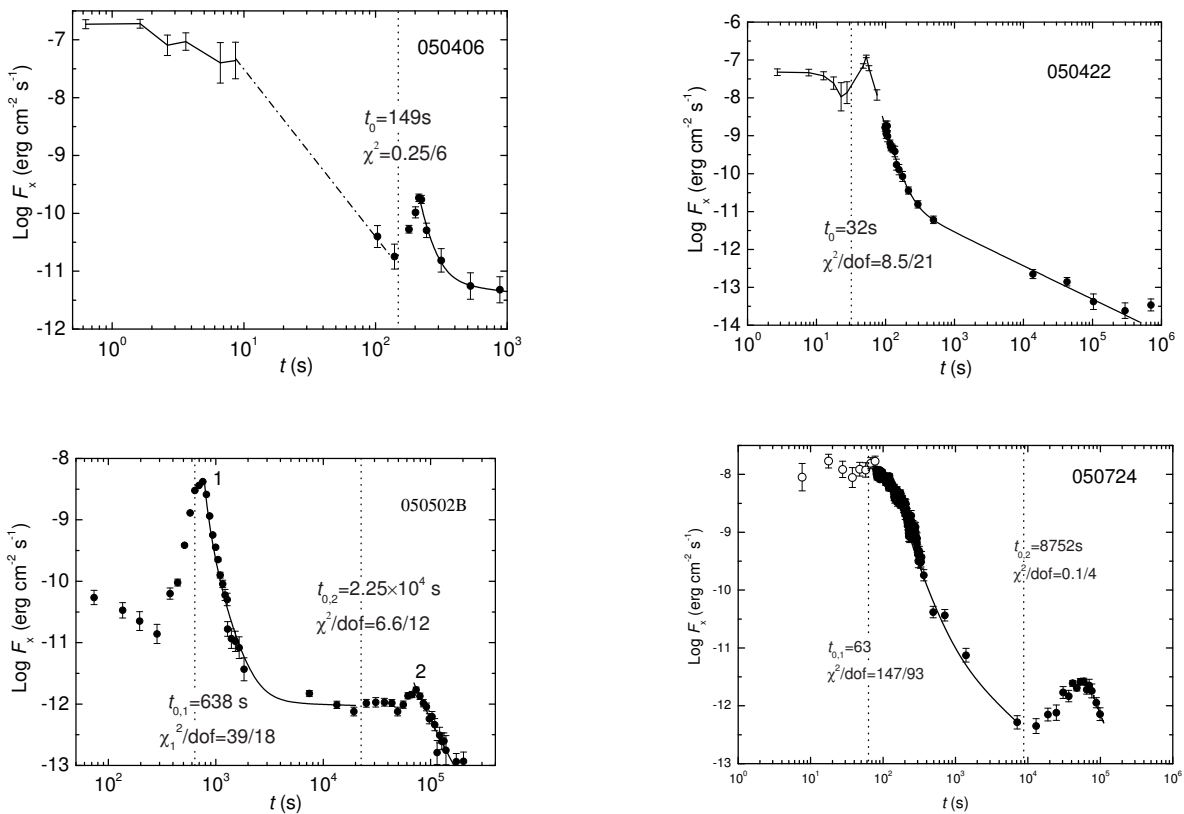

FIGURE 2. Several examples of the searched $t_{0}$ of X-ray flares. From Liang et al. [8].

gives the best fit to the data. The results are impressive. In many cases, the searched $t_{0}$ is exactly where it is expected, i.e. at the beginning of the corresponding flare (Fig.2). This gives strong support to our beginning hypothesis: that the flares are from internal dissipation of energy and that the decay is controlled by the curvature effect. In some cases the signature is not as clear as expected, but these cases also have overlapping flares or extended emission so that the rising part of the flare is usually burried beneath other emission components.

One interesting thing to note is that both GRB 050502B (a long-duration burst [6]) and GRB 050724 (a short-duration burst associated with non-star-forming galaxy [10]) have a late flare peaking around a day. The decay after the peak clearly follows the prediction of curvature effect, strongly suggesting that the central engine is turned on again (for an extended emission period) at such a late epoch. This poses great challenge to the possible central engine models for both long and short GRBs.

\section{HOW TO RESTART THE CENTRAL ENGINE}

Now that the X-ray flare data suggest that the GRB central engine must be re-started at a delayed time and that it works in a non-continuous manner, a straightforward question is then how a GRB central engine model would be able to give rise to what is observed.

This is still largely an open issue. Nonetheless a list of suggestions have been made since the discovery of the X-ray flares.

- King et al. [24] suggest that within the collapsar progenitor model for long GRBs, 
the rotating stellar core may undergo fragmentation during the collapse. The accretion of the fragmented blobs into the newly formed black hole offers a plausible mechanism of X-ray flares. Whether such a fragmentation can be realized calls for proofs from numerical simulations. The existence of similar X-ray flares in short GRB 050724 [10] suggests that the flare mechanism likely also work for progenitors without a heavy envelope. This makes this suggestion inconclusive.

- Based on energetics alone, Fan et al. [25] argue that the flares following the short GRB 050724 (and possibly also GRB 050709) must be of magnetic origin. This is because in various compact star merger models, the mass of the fuel to power the accretion is limited to be at most about 1 solar mass. The durations of the early flares typically last for hundreds seconds, suggesting that the accretion rate $\left(\leq 0.01 \mathrm{M}_{\odot} / \mathrm{s}\right)$ is too low for the neutrino-annihilation mechanism to power the observed $10^{48} \mathrm{erg} \mathrm{s}^{-1}$ luminosity. As a result, the X-ray flares are expected to be somewhat linearly polarized, although the detailed degree of polarization is hard to predict. The argument may be also extended to flares in long GRBs

- Motivated by the similarity of the X-ray flare properties for both long and short bursts, Perna et al. [16] argue that the flaring mechanism must be related to the property of something in common for both types of bursts. They suggest the accretion disk is this common link, and the episodical flares may be caused by fragmentation of the accretion disk itself. Gravitational instability in the outer parts of the disk may cause the fragmentation. The model can naturally interpret some properties of the flares, including the duration-timescale correlation and the duration-peak luminosity anticorrelation.

- Based on results of previous MHD numerical simulations and theoretical analyses, Proga \& Zhang [26] suggest that the accretion flow does not have to be chopped at large radii due to fragmentation. Rather, strong magnetic fields near the black hole can play the role to modulate the accretion flow. The magnetic barrier, which has been evident in previous numerical simulations [27, 28], can act as an agent to repeatedly stop and restart the accretion flow. This gives a plausible mechanism to power X-ray flares in both long and short GRBs. In this model, the launched jet is magnetized, which coincides the argument based on energetics [25].

- Dai et al. [29] argue that the postmerger product of double neutron star merger may well be a massive neutron star with milliseocnd rotation period. The differentiallyrotating stellar body would wind up interior poloidal magnetic fields to form progressively stronger toroidal magnetic fields. These tangled fields then float up and break through the stellar surfaces, triggering magnetic-reconnection-driven explosive events. This is an attractive mechanism to produce X-ray flares in short GRBs. A less specified central engine model with the similar idea is discussed in [30].

Entering the second-year operation, Swift will keep collecting flare data for a large sample of bursts. From data point of view, it is high time to systematically analyze the flare data and perform statistical analyses of the properties of flares. In the mean time, detailed theoretical modeling of the flare properties (e.g. [31]) would shed more light on the radiation mechanism and the dissipation site of X-ray flares. In long terms, coordinated observations of Swift and high energy detectors such as GLAST would 
reveal whether there are high-energy counterparts of X-ray flares, and hence, greatly constraint the emission physics of the flares [32]. An even far-reaching goal is to detect polarization of X-ray flares with future X-ray polarimeters, which would lead to more direct diagnoses of the GRB central engine [25].

\section{ACKNOWLEDGMENTS}

I thank stimulative collaborations with E. W. Liang, Y. Z. Fan, J. Dyks, D. N. Burrows, S. Kobayashi, P. Mészáros, R. Perna, P. J. Armitage, D. Proga, Z. G. Dai, X. Y. Wang, X. F. $\mathrm{Wu}$, J. Nousek, N. Gehrels and many other members of the Swift team on various topics covered in this talk. This work is supported by NASA through grants NNG05GB67G, NNG05GH91G, and NNG05GH92G.

\section{REFERENCES}

1. J. A. Nousek, C. Kouveliotou, D. Grupe, et al. Astrophys. J., in press (2006), astro-ph/0508332

2. B. Zhang, Y. Z. Fan, J. Dyks, S. Kobayashi, P. Mészáros, D. N. Burrows, J. A. Nousek, and N. Gehrels, Astrophys. J., in press (2006), astro-ph/0508321

3. G. Chincarini, A. Moretti, P. Romano, et al. astro-ph/0506453

4. P. T. O'Brien, R. Willingale, J. Osborne, et al. Astrophys. J., submitted (2006), astro-ph/0601125

5. D. N. Burrows, P. Romano, A. Falcone, et al. Science, 309, 1833 (2005).

6. A. Falcone, D. N. Burrows, D. Lazzati, et al. Astrophys. J., in press (2006), astro-ph/0512615

7. P. Romano, A. Moretti, P. L. Banat, et al. Astron. Astrophys., in press (2006), astro-ph/0601173

8. E. W. Liang, B. Zhang, P. T. O'Brien, et al. Astrophys. J., submitted (2006).

9. D. N. Burrows, P. Romano, O. Godet, et al. "Swift XRT Observations of X-ray flares in GRB afterglows", to appear in X-Ray Universe 2005, conference proceedings, (2005), astro-ph/0511039

10. S. D. Barthelmy, G. Chincarini, D. N. Burrows, et al. Nature, 438, 994 (2005).

11. S. Campana, G. Tagliaferri, D. Lazzati, et al. Astron. Astrophys., submitted (2006).

12. K. Ioka, S. Kobayashi, B. Zhang, Astrophys. J, 631, 429 (2005)

13. S. Kobayashi, B. Zhang, P. Mészáros, D. N. Burrows, Astrophys. J., submitted (2005), astro-ph/0506157

14. L. Piro, M. De Pasquale, P. Soffitta, et al. Astrophys. J., 623, 314 (2005)

15. A. I. MacFadyen, E. Ramirez-Ruiz, W. Zhang preprint, (2005), astro-ph/0510192

16. R. Perna, P. J. Armitage, B. Zhang, Astrophys. J., 636, L29 (2006)

17. Y. Z. Fan, D. M. Wei, Mon. Not. R. Astron. Soc., 364, L42 (2005)

18. B. Zhang, P. Mészáros, Astrophys. J., 566, 712

19. C. D. Dermer, in preparation (2006)

20. P. Kumar, A. Panaitescu, Astrophys. J., 541, L51 (2000)

21. C. D. Dermer, Astrophys. J., 614, 284 (2004)

22. A. Panaitescu, P. Mészáros, N. Gehrels, D. Burrows, J. Nousek, Mon. Not. R. Astron. Soc.. in press (2006), astro-ph/0508340

23. J. Dyks, B. Zhang, Y. Z. Fan, Asttrophys. J., submitted (2006), astro-ph/0511699

24. A. King, P. T. O’Brien, M. R. Goad, J. Osborne, E. Olsson, K. Page, Astrophys. J., 630, L113 (2005)

25. Y. Z. Fan, B. Zhang, D. Proga, Astrophys. J., 635, L129 (2006)

26. D. Proga, B. Zhang, Astrophys. J., submitted (2006), astro-ph/0601272

27. D. Proga, Begelman, M. C. Astrophys. J., 592, 767 (2003)

28. I. V. Igumenshchev, R. Narayan, M. A. Abramowicz, Astrophys. J., 592, 1042 (2003)

29. Z. G. Dai, X. Y. Wang, X. F. Wu, B. Zhang, Science, 311, 1127 (2006)

30. W. H. Gao, Y. Z. Fan Astrophys. J., submitted (2006), astro-ph/0512646 
31. X. F. Wu, Z. G. Dai, X. Y. Wang, Y. F. Huang, L. L. Feng, T. Lu, Astrophys. J., submitted (2006) astro-ph/0512555

32. X. Y. Wang, Z. Li, P. Mészáros, Astrophys. J., submitted (2006), astro-ph/0601229 